\title{
Anti-Glomerular Basement Membrane Disease Following Nephrectomy
}

\author{
Nicole Droz MD and Rula A Hajj-Ali MD ${ }^{1}$ \\ ${ }^{1}$ Cleveland Clinic Foundation, Department of Rheumatology and Immunology \\ ${ }^{*}$ Corresponding author: Dr. Nicole Droz, MD, Department of Rheumatologic and Immunologic Disease, Cleveland Clinic Foundation, Cleveland, Ohio, USA; TEL: \\ 216.444.5632; FAX: 2164457569; EMAIL: DROZN@ccf.org
}

Received: December 17, 2019; Accepted: December 18, 2019; Published: December 25, 2019

\begin{abstract}
A 51 year old female presented with rapidly progressive renal failure and diffuse alveolar hemorrhage following nephrectomy for retroperitoneal fibrosis. Anti-glomerular basement membrane (anti-GBM) antibodies returned strongly positive confirming a diagnosis of anti-GBM disease. She was treated with corticosteroids, plasma exchange and cyclophosphamide. To our knowledge, this is the first adult case of anti-GBM disease following nephrectomy.
\end{abstract}

Keywords: Vasculitis, Anti-GBM disease, nephrectomy

\section{Introduction}

Anti-Glomerular Basement Membrane (anti-GBM) disease is characterized by rapidly progressive glomerular nephritis with or without pulmonary hemorrhage [1]. It is usually monophasic in nature and disease severity correlates with antibody titer [1].Despite the known pathogenicity of anti-GBM antibodies, and the correlation of disease severity with their titers, the underlying pathogenesis of disease remains unclear. Here we describe a patient who developed anti-GBM disease following nephrectomy.

\section{Case Report}

A 51 year old female presented for a second opinion to our facility. She had a past medical history of seronegative rheumatoid arthritis which was diagnosed in early 2013 after she presented with joint pain following a personal stressor. She was treated with methotrexate and adalimumab with resolution of her presenting complaints. Three years later, during routine laboratory monitoring for her methotrexate, she was found to have an acute rise in her creatinine to $6.44 \mathrm{mg} / \mathrm{dL}$ above her baseline of around $1.0 \mathrm{mg} / \mathrm{dL}$. She reported some associated symptoms of malaise and abdominal pain. She was admitted to a local hospital for workup of her acute kidney injury. At that time, urinalysis was unremarkable and serologies for Antineutophilic Cytoplasmic Antibodies (ANCA) were negative. She did have elevated inflammatory markers (ESR $72 \mathrm{~mm} / \mathrm{hr}$ and CRP 18.7). CT of the abdomen and pelvis was performed and demonstrated severe, long-standing hydronephrosis of the right kidney and moderate hydronephrosis of the left kidney with associated hydroureters bilaterally. There was an amorphous $5.5 \times 2.8 \mathrm{~cm}$ soft tissue mass surrounding the aorta which was obstructing both ureters (Figure 1). The findings were consistent with retroperitoneal fibrosis. She underwent ultrasound guided bilateral percutaneous nephrostomy tube and stent placement with improvement of her creatinine to $3.0 \mathrm{mg} / \mathrm{dL}$. Percutaneous biopsy performed at the time of the procedure confirmed the diagnosis of retroperitoneal fibrosis with negative staining for IgG4.

She was referred to our center for second opinion. During her evaluation, her right kidney was determined to be non-functioning. Urology recommended right sided nephrectomy and left sided ureterolysis and stent placement. Her post-operative course was uncomplicated and she was discharged on hospital day three. Histopathologic examination of the right kidney revealed interstitial fibrosis with tubular atrophy and moderate arteriolosclerosis consistent with her history of chronic obstructive nephropathy. Two weeks following discharge, she was re-admitted for symptoms of cough, dyspnea and oliguria. She was afebrile and normotensive but was tachycardic. She was tachypneic with a respiratory rate of 41 and oxygen saturation of $91 \%$ on $50 \%$ hi-flow nasal cannula. Her physical examination revealed bilateral coarse crackles throughout both lung fields. She had no rashes, sinus abnormalities or musculoskeletal abnormalities and the rest of her examination was unremarkable. Her laboratory evaluation revealed a rise in creatinine to $7.8 \mathrm{mg} / \mathrm{dL}$. Her hemoglobin declined to 8.6 from her baseline of 10.6. Inflammatory markers were again elevated. Her urinalysis did show a large amount of blood as well as a moderate amount of leukocytes. Urine culture later grew E. faecalis. She was treated for sepsis secondary to urinary tract infection with vancomycin and levofloxacin but failed to have improvement in her kidney function and quickly became anuric and required dialysis.

During this time, she became progressively more hypoxemic requiring supplemental oxygen by high flow nasal cannula. CT of the chest demonstrated bilateral ground glass opacities (Figure 2). Bronchoscopy with BAL was performed demonstrating progressively more bloody aliquots indicative of diffuse alveolar hemorrhage. 
Workup for infectious etiologies was negative. Further serologic workup was obtained to assess for systemic diseases. ANCAs were again negative, butanti-GBMantibodies were strongly positive at a titer of $>200 \mathrm{RU} / \mathrm{ml}$. The patient was diagnosed with anti-GBM disease based on her clinical presentation and positive anti-GBM antibodies. A repeat renal biopsy was deferred due to her solitary kidney status.
She was treated with corticosteroids, cyclophosphamide and plasma exchange with improvement in her pulmonary manifestations but unfortunately never achieved renal recovery and is awaiting renal transplantation. In the setting of her previously unremarkable renal biopsy, we speculate that her urologic procedure may have led to the development of anti-GBM disease.

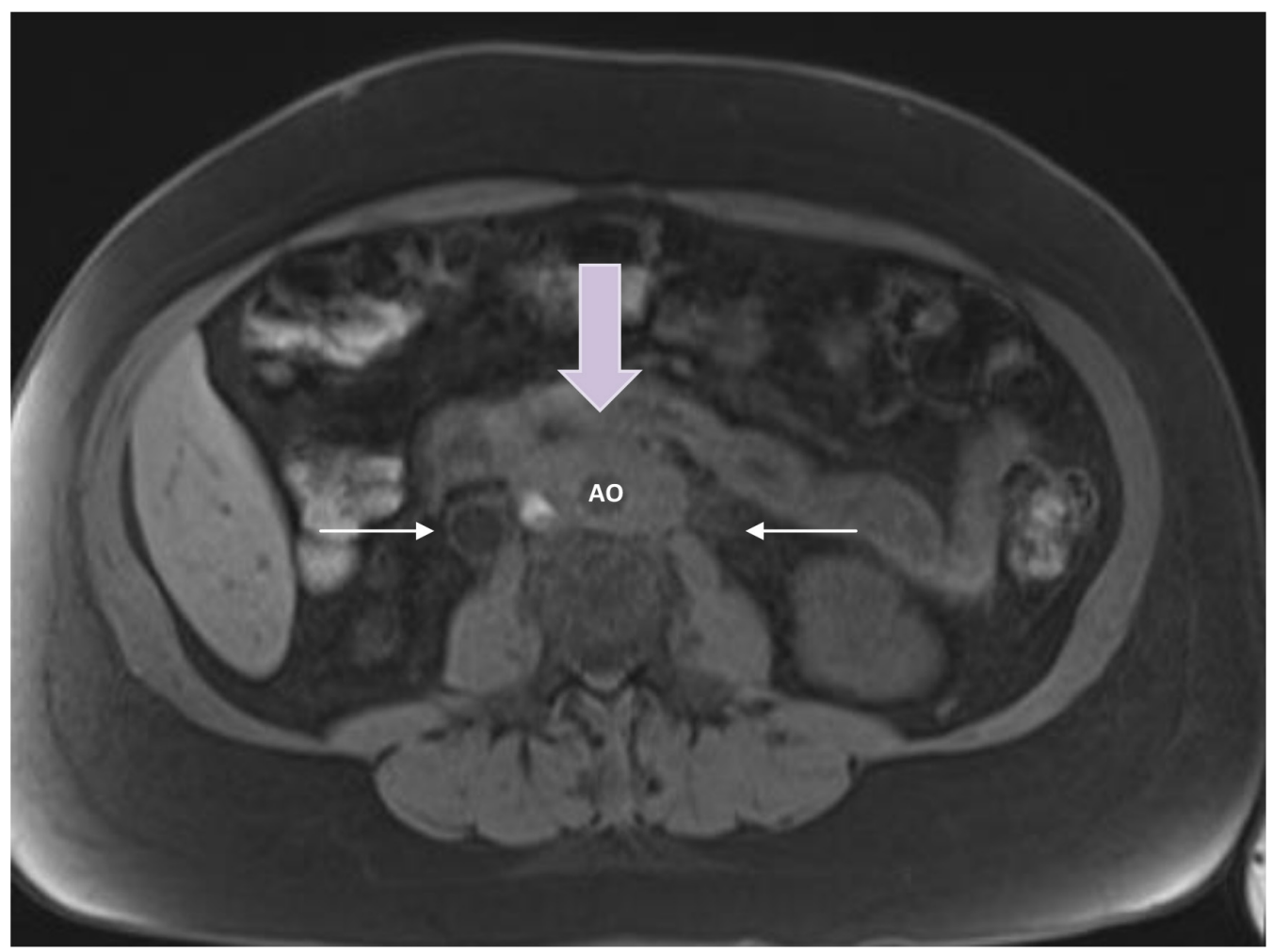

Figure 1. Retroperitoneal mass encasing the aorta with associated hydro-ureters. Yellow arrow = retroperitoneal mass; white arrows = ureters; $\mathrm{AO}=$ aorta

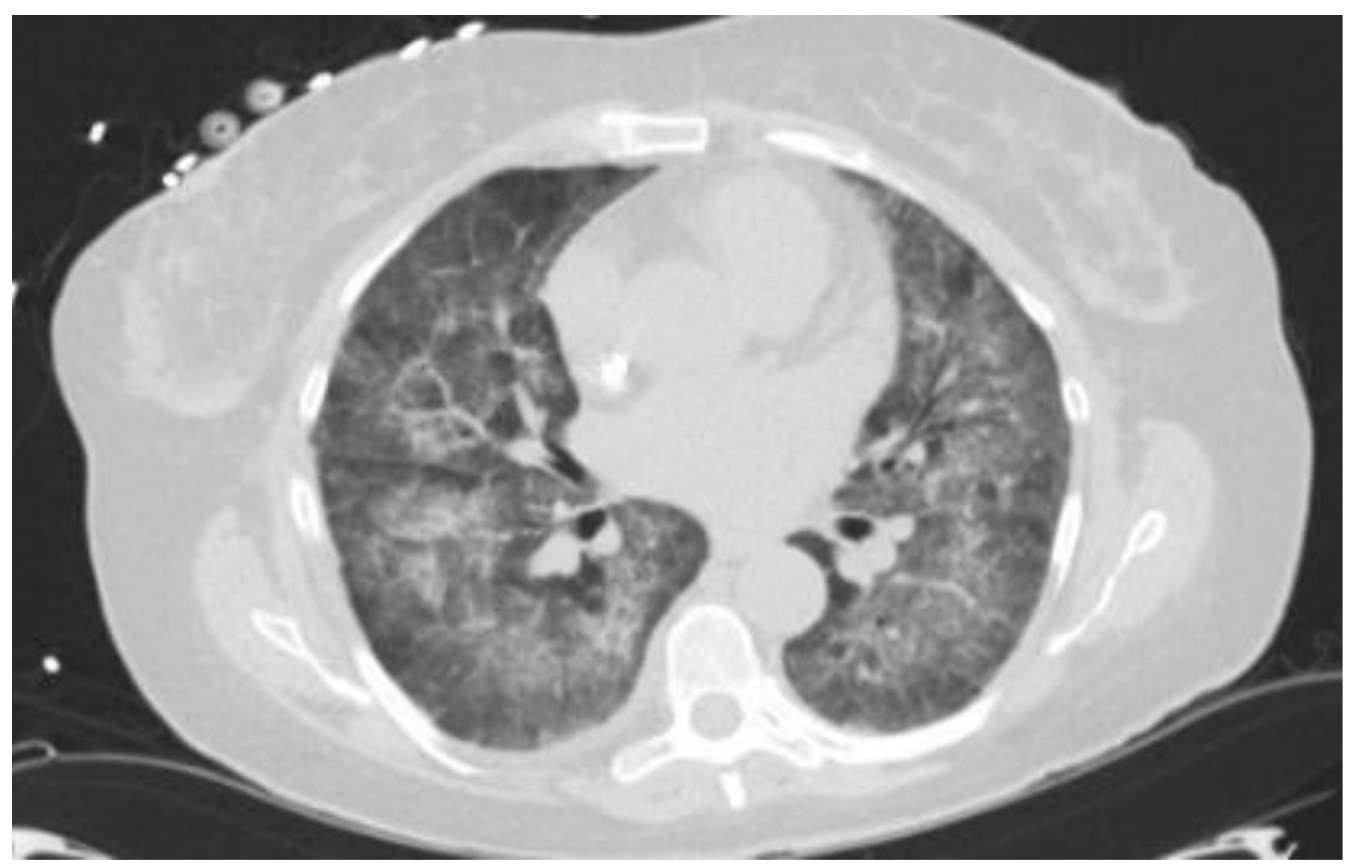

Figure 2. CT of the chest demonstrating bilateral ground glass opacities 


\section{Discussion}

Anti-GBM disease is characterized by rapidly progressive glomerular nephritis with or without pulmonary hemorrhage. The disease is mediated by pathogenic autoantibodies directed against the non-collagenous domain of the a 3 chain of type IV collagen. Antibodies are generally IgG, but IgA and IgM have also been reported [1]. Although it is well known that the antibodies are pathogenic, the underlying pathogenesis of disease has not been elucidated. There is a strong HLA association with the disease with an over representation of HLA-DR15 and HLA-DR4 alleles suggesting a possible genetic component. Further, HLA-DR7 and HLA-DR1 seem to be protective as they are underrepresented in disease [2]. Others have postulated that because of the cryptic nature of the antigenic target in antiGBM disease, disruption of collagen hexamer is necessary to initiate disease [3]. Anti-GBM disease has been reported after environmental exposures such as tobacco use or hydrocarbons, bacterial or viral infections and from other glomerular diseases like ANCA associated vasculitis and membranous nephropathy [4- 6]. These exposures may alter the configuration of the collagen hexamer exposing the cryptic antigen leading to disease.

There have also been reports of onset of Anti-GBM disease following macroscopic renal damage. To investigate this mechanism further, authors Takeuchi et al hypothesized that mechanical renal damage may be necessary for exposure of the cryptic antigen to induce antibody production. In their retrospective study, they evaluated patients who had anti-GBM antibodies done at the time of diagnosis of hydronephrosis. They identified 11 patients for inclusion into their study. 3 of these patients had elevated anti-GBM antibody titers. In 1 patient, anti-GBM antibody levels returned to normal after treatment of hydronephrosis [7]. This study, although small, lends support to the paradigm that disruption of the collagen hexamer and cryptic antigen exposure is necessary to induce disease. Surgical procedures, such as lithotripsy, have also been implicated in provoking the disease [8-10]. To our knowledge, this is the first case of an adult patient diagnosed with Anti-GBM disease following nephrectomy. Authors Hagan et al previously reported the first pediatric patient to present with Anti-GBM disease following nephrectomy for xanthogranulomatous pyelonephritis [11]. Our case report lends credence to the hypothesis that cryptic antigen exposure is necessary for Anti-GBM disease development. Although rare, Anti-GBM disease should be suspected in patients who presents with rapidly progressive glomerulonephritis with or without pulmonary hemorrhage following urologic procedures. Early recognition is critical for prompt treatment and improved patient outcomes.

\section{References}

1. Salama A, Levy J, Lightsone L (2001) Goodpasture's disease. Lancet 358: 917-920.

2. Fisher M, Pusey CD, Vaughan RW, Rees AJ (1997) Susceptibility to anti-glomerular basement membrane disease is strongly associated with HLA-DRB1 genes. Kidney Int 51: 222-229. [crossref]

3. Pedchenko V, Bondar O, Fogo A, Vanacore R, Voziyan P et al. (2010) Molecular Architecture of the Goodpasture Autoantigen in Anti-GBM nephritis. N Engl J Med 363: 343-354. [crossref]

4. Wilson C, Smith R (1972) Goodpasture's syndrome associated with influena A2 virus infection. Ann Intern Med 76: 91-94. [crossref]
5. Beirne G, Brennan J (1972) Glomerulonephritis associated with hydrocarbon solvents. Archives of Environmental Health: An International Journal 25: 365-369.

6. Kitagawa W, Miura N, Yamada H, Nishikawa K, Futenma A et al. (2005) The increase of antiglomerular basement membrane antibody following pauci-immunetype crescentic glomerulonephritis. Clin Exp Nephrol 9: 69-73. [crossref]

7. Takeuchi Y, Takeuchi E and Kamata K (2015) A possible Clue for the Production of Anti-Glomerular Basement Membrane Antibody Associated with Ureteral Obstruction and Hydronephrosis. Case Rep Nephrol Dial 5: 87-95. [crossref]

8. Guerin V, Rabian C, Noel LH (1990) Anti-Glomerular basement membrane disease after lithotripsy. Lancet 335: 856-857.

9. Xenocostas A, Jothy S, Collins B, Loertscher R, Levy M (1999) Anti-glomerular basement membrane glomerulonephritis after extracorporeal shock wave lithotripsy. Am J Kidney Dis 33: 128-132. [crossref]

10. Umekawa T, Kohri K, Iguchi M, Yoshioka K, Kurita T (1993) Glomerular Basement membrane antibody and extracorporeal shock wave lithotripsy. Lancet 341: 556. [crossref]

11. Hagan E, Mallett T, Convery M, McKeever K (2015) Anti-GBM disease after nephrectomy for xanthogranulomatous pyelonephritis in a patient expressing HLADR15 major histocompatibility antigens: a case report. Clin Nephrol 3: 25-30. [crossref]
Citation:

Nicole Droz \& Rula A Hajj-Ali (2019) Anti-Glomerular Basement Membrane Disease Following Nephrectomy. Integr J Nephro Urol Stud Volume 1(1): 1-3. 